\title{
Improved solution for laminar thermal boundary layers
}

\author{
BY
}

Enzo O. Macagno, Matilde Macagno

AND

Touvia Miloh

Institute of Hydraulic Research of the University of Iowa

Institut für Hydromechanik, Universität Karlsruhe

and School of Engineering, Tel-Aviv University

\section{Introduction}

The equations governing the temperature distribution in the neighborhood of a flat plate are quite sensitive to the accuracy attained in the calculation of the velocity field. This is due, among other things, to the role played by the second derivative of the stream function in the process of integration to obtain the temperature distribution. In the now classical temperature profiles of Polhausen's special solutions it was not possible to detect inconsistencies, but they appeared in plots of the general solution. The motivation for this work stems from the discovery of such inconsistencies by students of one of the authors, as described in the acknowledgements.

This paper presents an analysis of the general properties of the governing equations for forced laminar flow past flat plates parallel to the flow at infinity. Heat generated by viscous dissipative terms is taken into account, but the pressure gradient is assumed to be zero, and the effect of buoyancy to be negligible. Calculations were performed with special care in order to eliminate spurious trends in the temperature distributions. Graphs are presented to illustrate the solutions thus obtained, and tables are available on request.

\section{Background and qualitative analysis}

A little over fifty years ago, the first calculations of thermal boundary layers were published by E. Pohlhausen [1], who considered two fundamental cases. The first case was that of a heated plate with uniform temperature in the midst of an incompressible fluid with uniform velocity and temperature at infinity. The second case was that of an adiabatic plate also in a steady uniform flow of a fluid with uniform temperature. In a literature search, no previous work could be found, except for an analysis by L. Prandtl [2] in which no calculations of temperature distributions were included. Pohlhausen's analysis has become a classic of boundary layer methodology, but, for the calculations of temperature distributions, he used the data available at the time for the velocity distribution in the boundary layer [3]. This did not affect the expected qualitative trend of the temperature profiles which he determined for the two particular cases mentioned above. It is only when Pohlhausen's results are used to construct the general solution for the thermal boundary layer that the inaccuracies in the velocity profiles are large enough to distort some of the temperature profiles [4]. This occurs in a manner that can be easily detected as anomalous even by a relatively inexperienced observer.

To facilitate the discussion, we summarize first the classical treatment of the problem, and then show how possible wrong trends can be detected through a simplified qualitative analysis. This is accomplished by establishing basic properties that the solution must possess. The governing equations for laminar flat-plate boundary layers without pressure gradient and buoyancy effects, but with dissipation of energy, are :

$$
\begin{gathered}
\frac{\partial u}{\partial x}+\frac{\partial v}{\partial y}=0 \\
\rho\left(u \frac{\partial u}{\partial x}+v \frac{\partial u}{\partial y}\right)=\mu \frac{\partial^{2} u}{\partial y^{2}}
\end{gathered}
$$




$$
\rho c_{p}\left(u \frac{\partial T}{\partial x}+v \frac{\partial T}{\partial y}\right)=k \frac{\partial^{2} T}{\partial y^{2}}+\mu\left(\frac{\partial u}{\partial y}\right)^{2}
$$

The first of these equations is the equation of continuity for the velocity vector $(u, v)$ in the two-dimensional space $(x, y)$. The second is the momentum equation, and the third is the energy equation, both simplified according to Prandtl's approximation for flat-plate boundary layers. The physical properties, density $\rho$, viscosity $\mu$, specific heat $c_{p}$, and thermal conductivity $k$, are assumed to be constant. For this, the temperature $T$ must vary within a relatively narrow interval.

The system (1)-(3) can be reduced to a system of two equations if, following Blasius [3], the Lagrangian stream function $\psi$ is introduced. The treatment of equations (1)-(3) is classical and only the final dimensionless forms of the equations will be given here. We have :

$$
\begin{gathered}
f f^{\prime \prime}+2 f^{\prime \prime}=0 \\
T^{\prime \prime}+(1 / 2) P f T^{\prime}=-1 / 2 \frac{P U_{\alpha}^{2}}{c_{p}} f^{\prime \prime \prime}
\end{gathered}
$$

where

$$
\begin{aligned}
& f(\eta)=\psi / \sqrt{v x U_{*}} \\
& \eta=y\left(U_{\infty} / v x\right)^{1 / 2}
\end{aligned}
$$

and

$$
P=\mu c_{p} / k
$$

is the Prandtl number. The symbol $v$ denotes the kinematic viscosity of the fluid. Once the function $f$ is determined by integration of equation (4), the velocity components can be obtained in the manner given by Blasius. When the temperature distribution is desired, one can directly use the function $f$ in equation (5), and proceed to integrate this equation.

In mathematical terms, Pohlhausen proceeded in the following way. He found first the solution of the homogeneous equation for equation (5), and then a particular solution for the complete equation (5). Those two solutions are indicated with $\theta_{1}(\eta)$ and $\theta_{2}(\eta)$. The general solution for arbitrary wall temperature $T_{\text {"r }}$ was obtained by superposition:

$$
T-T_{*}=c \theta_{1}(\eta)+y_{2} \frac{U^{2}}{c_{p}} \theta_{2}(\eta)
$$

herein:

$$
c=T_{u r}-T_{x}-1, \frac{U^{2} x_{x}}{c_{p}} \theta_{2,}(0)
$$

In dimensionless form we have:

$$
\begin{array}{r}
\theta_{1}(n, P, F)=\frac{T-T_{x}}{T_{1}-T_{x}}=(1-F) \theta_{1}(\eta, P) \\
+F \theta_{3}(\eta, P)
\end{array}
$$

where $F=1 \leqslant L b(P)$ :

$$
\theta_{33}(\eta, P)=\frac{\theta_{, 2}(\eta, P)}{b(P)}
$$
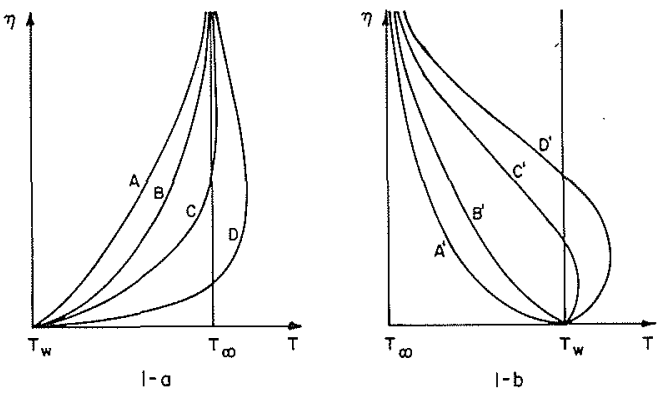

$1 /$ Illustrative temperature profiles for cool plate (1-a) and warm plate $(1-b)$.

The Eckert number $E$ is here defined by:

$$
E=\frac{U^{2}}{c_{p}\left(T_{10}-T_{s}\right)}
$$

and $b(P)$, a function of the Prandtl number is defined by:

$$
b(P)=\theta_{2}(O, P)
$$

The functions $\theta_{1}$ and $\theta_{3}$ vary monotonically from 1 to 0 as the independent variable $\eta$ varies from 0 te $\infty$. Obviously, linear combinations of these two functions do not necessarily behave as monotonic functions.

Before we present a detailed analysis of the properties of the function $\theta_{4}$, we will discuss in a heuristic manner the shape that can be expected for the temperature profiles in laminar boundary layers in which the dissipation heat can produce notable shape distortions of those profiles. We will consider separately the cases of cool plates and warm plates

$$
\left(T_{r}<T_{r}, \quad T_{r r}>T_{r_{0}}\right)
$$

When the dissipation heat can be neglected, the temperature profiles for those two cases are like profiles $A$ and $A$ in Figs. 1-a and 1-b. Without loss of generality, we can assume that $T_{r}=0$ for the case plotted in Fig. $1-\mathrm{a}$, and that $T_{-}=0$ for the case shown in Fig. 1-b. If the effect of frictional heat is relatively small, profiles $A$
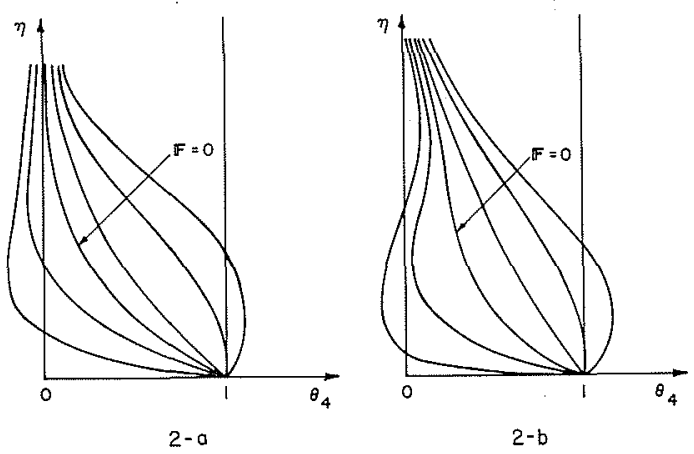

2/ Illustrative temperature profiles for the general case. Correct trend in 2-a, and erroneous trend in 2-b. (See Nomenclature for meaning of symbol F). 
and $A^{\prime}$ must appear slightly modified as illustrated by the curves $B$ and $B^{\prime}$, with heat flux still in the same directions as for $A$ and $A^{\prime}$. If the frictional sources of heat are much more productive, only part of the heat flux will be in the original directions, the rest will go towards the ambient fluid, as illustrated by profiles $C$ and $C^{\prime}$, and $D$ and $D^{\prime}$. Although not shown in the drawings, the shape of the function $\partial u / \partial y$ has been taken into account to locate the bulge of temperature near the location of the zone of maximum dissipation of energy. A similar qualitative analysis can be performed for an adiabatic plate. And then one can put together the general solution as a linear combination of simpler solution. This may lead to results like those in Figs. 2-a and 2-b; but one should be able to discard results like those in Fig. 2-b in which oscillatory behavior is noticed.

Temperature profiles should look like those in Fig. 2a and not like those in Fig. 2-b. This can be concluded from the fact that it is impossible for any temperature profile determined by equation (5) to have a minimum of $T$; only maxima are possible, although not necessary. This is immediately evident, from making $T^{\prime}=0$ in equation (5), to see what condition is obtained for points at which there is an extremum. We obtain

$$
T^{\prime \prime}{ }^{\prime \times 1 r}=- \text { const. }\left(f^{\prime \prime}\right)^{2}
$$

which corresponds to a maximum, not to a minimum. From a physical viewpoint, a minimum in any of the temperature profiles (Fig. 2-b) would correspond to a cool layer inserted between warmer ambient fluid and fluid warmed up by viscous dissipation. This is physically impossible in the present flow, given the condition of uniform temperature upstream from the plate.

\section{Quantitative analysis}

Taking into account Pohlhausen's solution for the function $\theta_{4}$ [4], the following expression can be adopted,

$$
\begin{aligned}
& \theta_{+}(\eta, P, E)=(1-F) \frac{\int_{\eta}^{\infty}\left[f^{\prime \prime}(\xi)\right]^{P} d \xi}{\int_{\eta}^{\infty}\left[f^{\prime \prime}(\xi)\right]^{P} d \xi} \\
& +2 F \frac{P}{b} \int_{\eta}^{\infty}\left[f^{\prime \prime}(\xi)\right]^{P}\left(\int_{0}^{\xi}\left[f^{\prime \prime}(\xi)\right]^{2 \ldots \prime^{\prime}} d \zeta\right) d \xi
\end{aligned}
$$

One can see that the solution depends on the second derivative of the Blasius function $f$. Since the values of the Prandtl number of usual interest may be large, errors may be important in the calculation of this function.

The boundary conditions are:

$$
\text { at } \eta=0 \text {, }
$$

$$
\theta_{1}=1 ; \quad \theta_{3}=0 ; \quad \theta_{4}=1
$$

at $\eta \rightarrow \infty$,

$$
\theta_{1}=0 ; \quad \theta_{3}=0 ; \quad \theta_{4}=0
$$

To study the possibility of extreme values of the function $\theta_{4}$ we take the derivative of this function and set it equal to zero:

$$
\begin{aligned}
& 1-F \\
& \quad+\frac{2 P F}{b}\left(\int_{0}^{\infty}\left[f^{\prime \prime}(\xi)\right]^{\prime \prime} d \xi\right)\left(\int_{0}^{\pi}\left[f^{\prime \prime}(\xi)\right]^{2-P} d \xi\right)=0
\end{aligned}
$$

If $F=1$ (or $b E=2$ ), the slope of the curve is zero at $\eta=0$ (adiabatic wall), since $f^{\prime} \geqslant 0$ for all $\eta$.

If we call $A(\eta)$ the function given by the product of the integrals in equation 18 we have :

$$
1-F+2 P F A(\eta)=0
$$

because $A(\eta) \geqslant 0$ for all $\eta$ and because $A(\eta)$ is monotonic there is only one value of $\|$ representing a solution of equation (19). In a more detailed manner we can show this by assuming that there is one extremum at $n_{1}$ and another at $\eta_{2}=\eta_{1}+\delta$, we have then :

$$
\begin{aligned}
& 1-F+2 P F A\left(\eta_{1}\right)=0 \\
& 1-F+2 P F A\left(\eta_{2}\right)==0
\end{aligned}
$$

From equation (20) we obtain :

$$
A\left(\eta_{1}\right)=A\left(\eta_{2}\right)
$$

or:

$$
\int_{-11}^{\eta_{1}}\left[f^{\prime \prime}(\zeta)\right]^{2 \cdots} \cdot d \zeta=\int_{-11}^{\eta_{1} \cdots \delta}\left[f^{\prime \prime}(\zeta)\right]^{2 \cdots \prime \prime} d \zeta
$$

and since $f^{\prime \prime}(\xi)$ is a continuous function, one gets:

$$
\int_{0}^{-\delta}\left[f^{\prime \prime}(\zeta)\right] \cdots \cdots l^{\prime} d \zeta=0
$$

Because $f^{\prime \prime}(\eta) \geqslant 0$ the only solution of equation (23) is $\delta=0$. Hence, only one extremum is possible, and this extremum is a maximum.

\section{Calculations and results}

The functions $\theta_{1}, \theta_{2}$, and $\theta_{i}$ were calculated by numerical methods subject to careful controls of their accuracy. Of course, these functions cannot be more accurate than the Blasius function for the flat-plate boundary layer. The tables by A. Smith [5] were chosen because they are highly accurate.

It was found during the calculations that the original expressions for $\theta_{2}$ and $\theta_{3}$ tended to generate inaccuracies for large values of the argument. To improve the ac- 


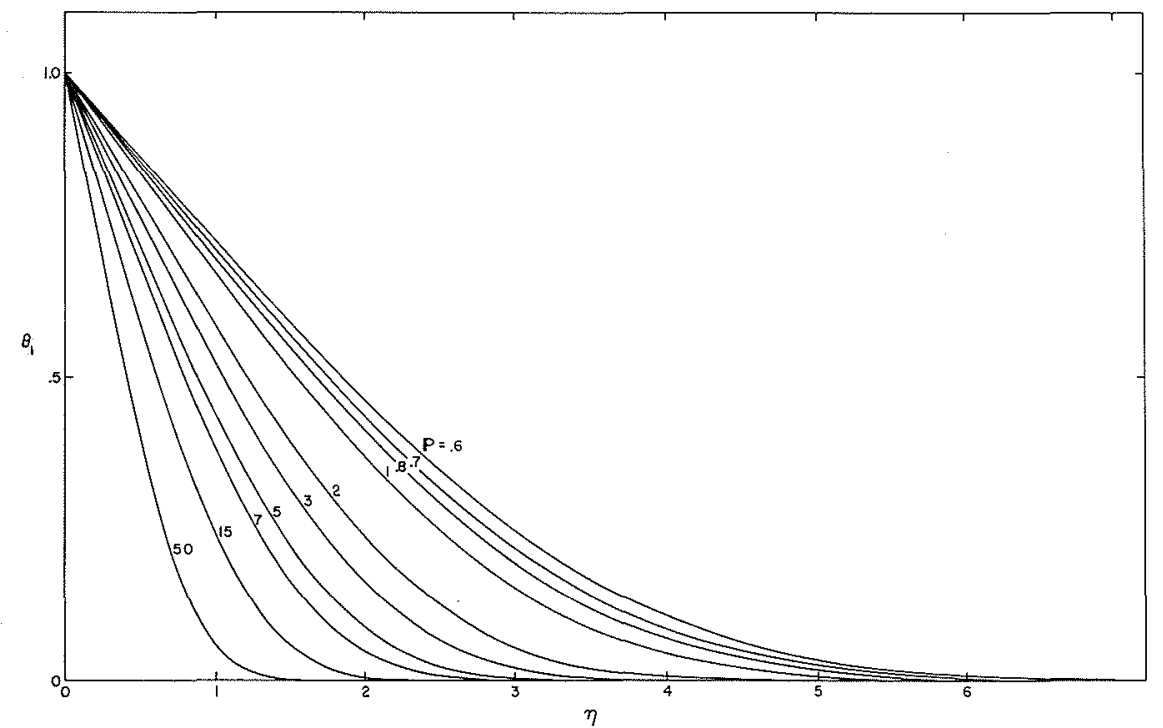

3/ Dimensionless temperature $\theta_{1}$ in terms of

dimensionless distance dimensionless distance
and Prandtl's number $P$.

curacy the expressions were transformed into the following ones:

$$
\theta_{1}(\eta, P)=1-\int_{0}\left[f^{\prime \prime}(\zeta)\right]^{\prime \prime} d \xi / \int_{0}^{\infty}\left[f^{\prime \prime}(\zeta)\right]^{\prime \prime} d \xi
$$

$$
\theta_{33}(\eta, P)=-\frac{\int_{0}^{\eta}\left[f^{\prime \prime}(\zeta)\right]^{2}\left(\int_{0}^{\zeta}\left[f^{\prime \prime}(\zeta) / f^{\prime \prime}(\xi)\right]^{\prime \prime 2} d \xi\right) d \zeta}{\int_{0}^{\infty}\left[f^{\prime \prime}(\zeta)\right]^{2}\left(\int_{0}^{\zeta}\left[f^{\prime \prime}(\zeta) / f^{\prime \prime}(\xi)\right]^{\prime \prime 2} d \xi\right) d \zeta}
$$

$$
\theta_{+}(\eta, P, F)=(1-F) \theta_{1}(\eta, P)-F \theta_{3}(\eta, P)
$$

Due to high powers in equation (25), calculations were performed in two steps to accomodate to compu- ter characteristics. This was done actually for all powers, i.e. for all Prandtl numbers. Simplicity in the calculations was ensured by using the trapezoidal rule but, of course, intervals were made small enough to obtain high accuracy. The step size was $\Delta \eta=0.05$ over the range $0 \leqslant n \leqslant 5.6$. The truncation error in the quadrature formula was estimated to be of the order of $2.10^{-6}$.

In Fig. 3, the temperature profiles near a heated flat plate for different Prandtl numbers ranging from 0.6 to 50 are presented. In this case, the heat due to viscous dissipation has been neglected. In Fig. 4, temperature profiles near an adiabatic flat plate are given. Figures 5 to 8 depict the temperature distributions given by the function $\theta_{f}$. These curves represent an improved result with respect to available curves (Schlichting [4], Parker et al. [6]). The dimensionless number $F$ varies from - 3 to 3 , while the Prandtl number takes the values $0.7,2.0,7.0$ and 15.0 .

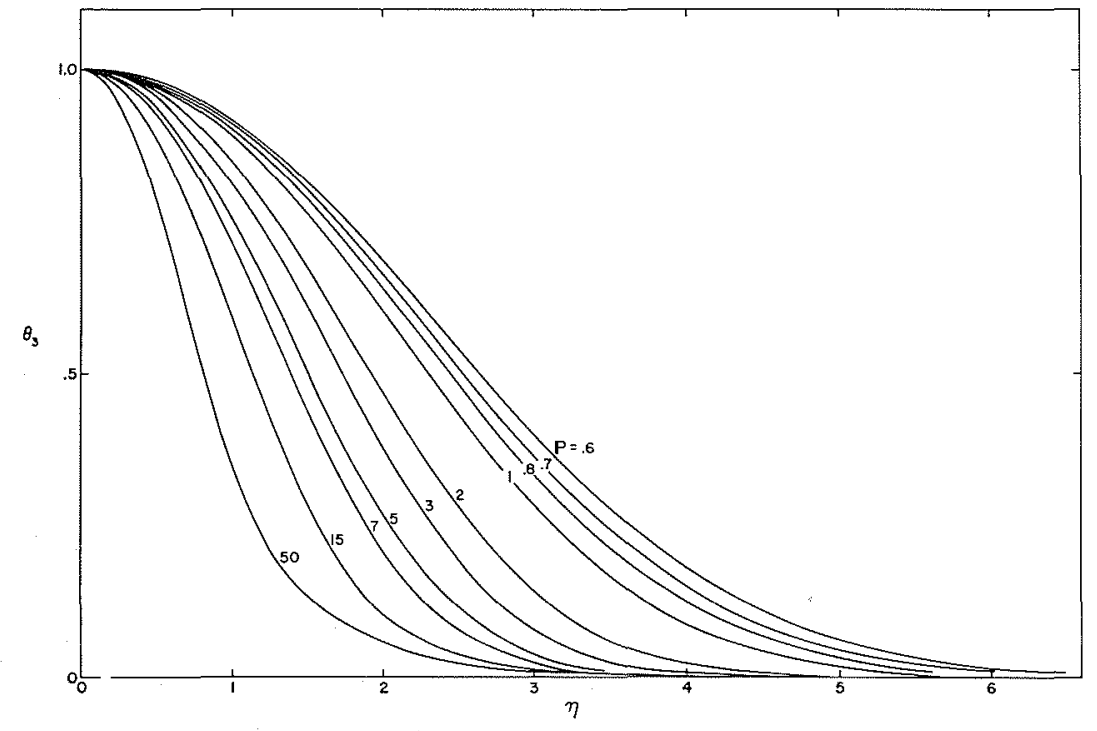

4/ Dimensionless temperature in terms of dimensionless distance and Prandtl's number $P$. 
5/ Dimensionless temperature $\theta_{\text {, }}$ in terms

of dimensionless

distance 1 and the

$F$ number,

for a PrandtI number

of 0.7 .

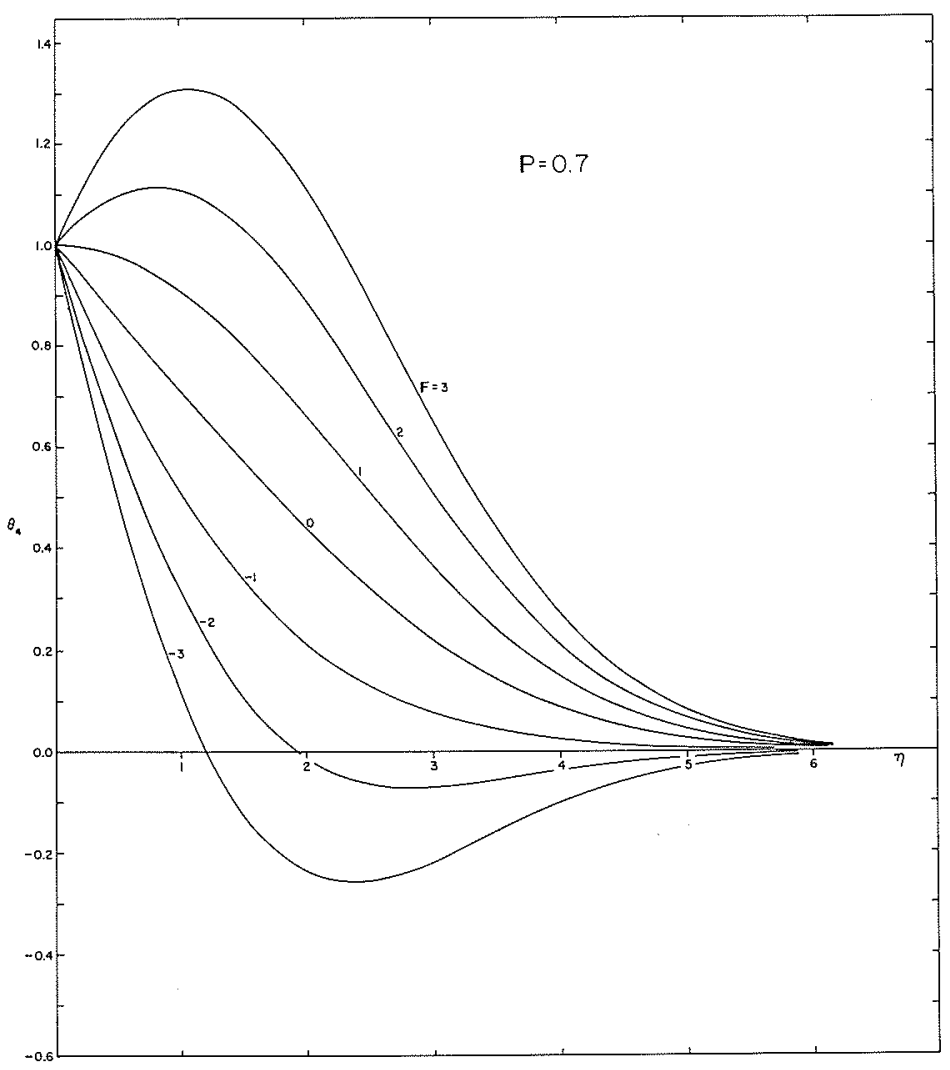

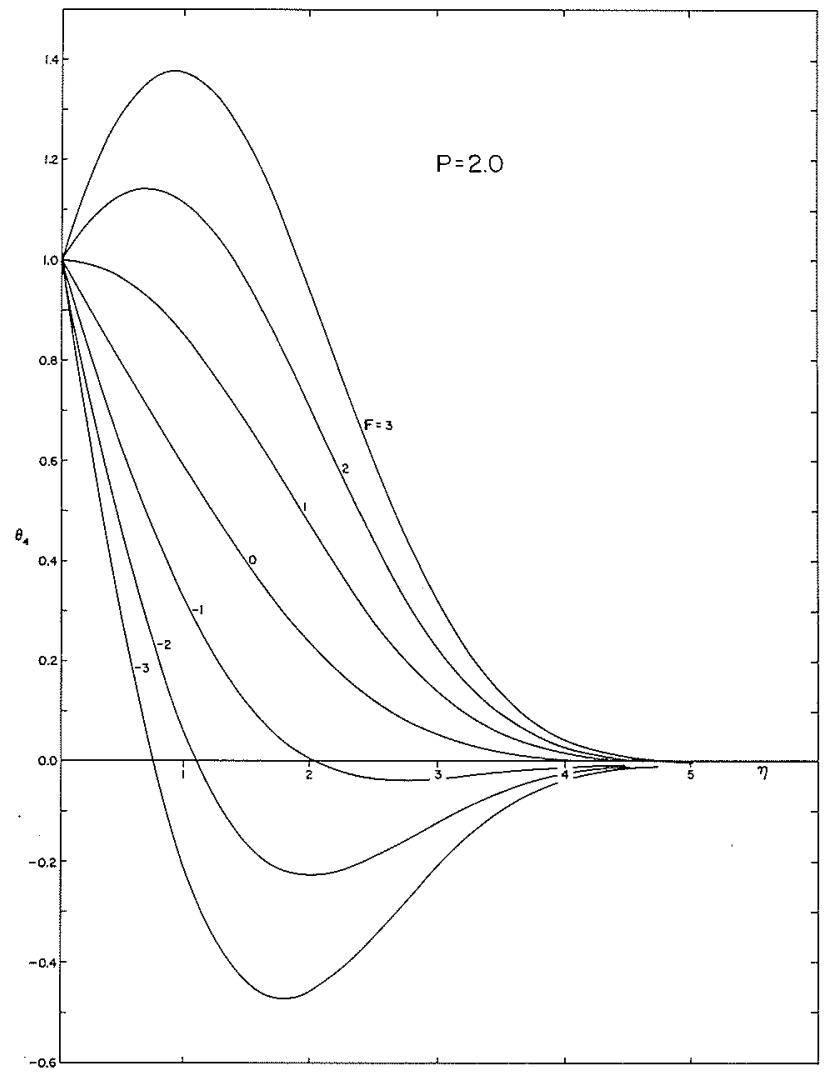

6/ Dimensionless temperature ${ }_{4}$ in terms of dimensionless distance $\eta$ and the $F$ number, for a Prandtl number of 2.0 .

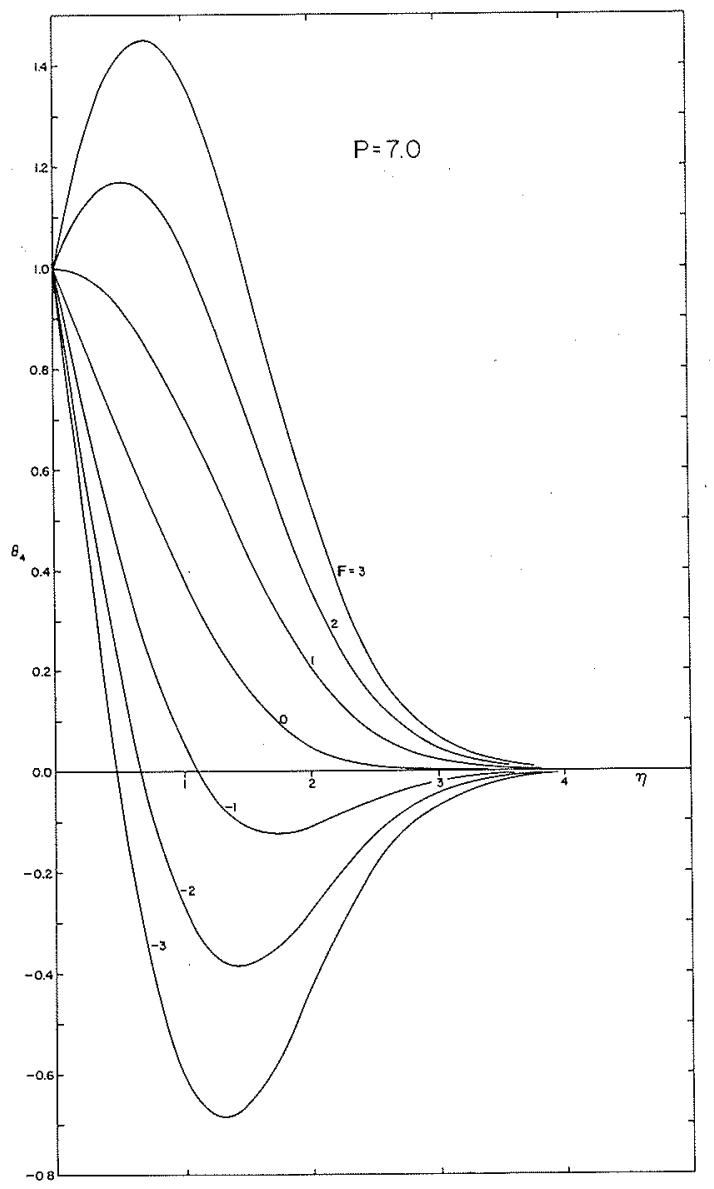

7/ Dimensioniess temperature $\hat{t}_{4}$ in terms of fimensionless distance $\eta$ and the $F$ number, for a Prandtl number of 7.0 . 


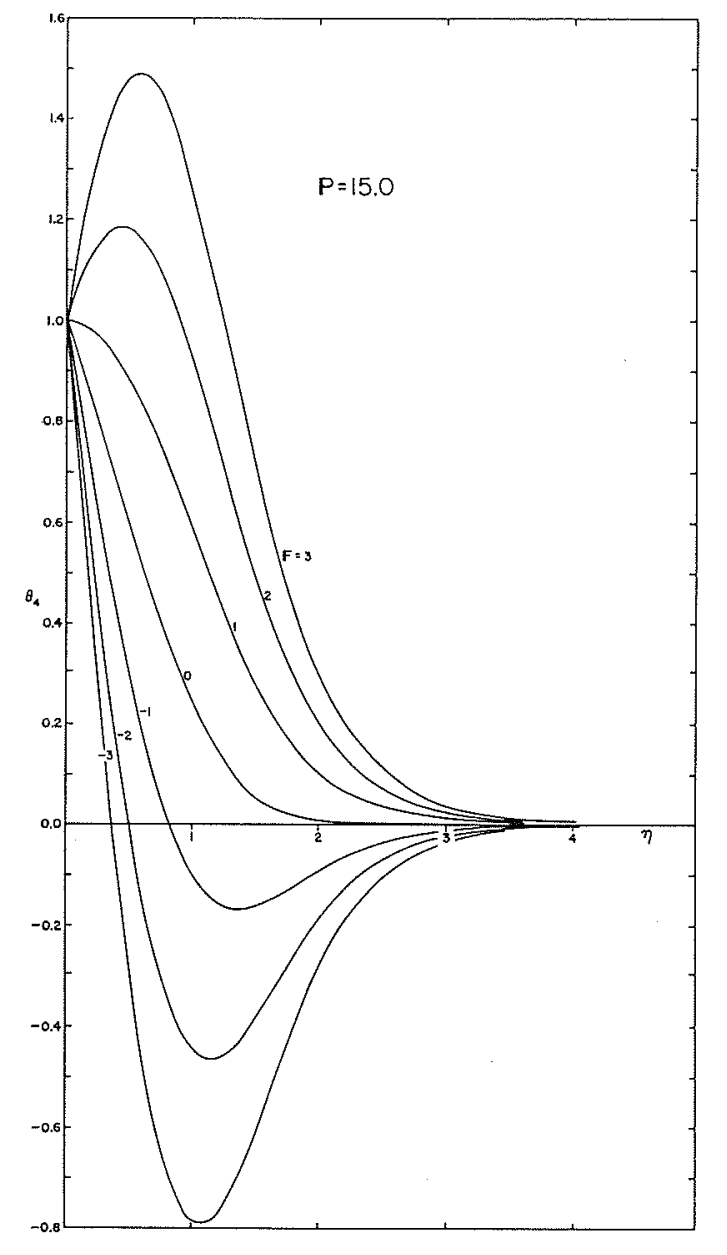

8/ Dimensionless temperature $\theta_{4}$ in terms of dimensionless distance $\eta$ and the $F$ number, for a Prandtl number of 15.0.

\section{Conclusion}

Existing curves for the temperature distribution in forced laminar boundary layers along flat plates at zero incidence show some anomalous temperature profiles which are physically and mathematically unaccepta- ble. More accurate methods were used to improve the functions originally presented by Pohlhausen, here denoted by $\theta_{1}$ and $\theta_{3}$. With the improved functions $\theta_{1}$ and $\theta_{3}$, the function $\theta_{4}$ for the general case has been recalculated more accurately. Graphs are given, and tables are available on request.

\section{Acknowledgements}

This research was partially supported by funds from the Office of Water Resources Research (Department of the Interior) (Grant B-041-IA). A contribition from the Graduate College of the University of Iowa is also acknowledged. Students of Intermediate Fluid Mechanics detected the anomalous trend, and one of them. Mr. Marco Roman, undertook independent calculations which were very useful to verify the numerical results of the authors.

\section{References}

[1] Pohlhausen (E.). - Der Wärmeaustauch zwischen festen Körpern mit kleiner Reibung und kleiner Wärmeleitung. Zeitschrift $f$. angew. Mathematik u. Mechanik, vol. I (1921).

[2] Prandtl (L.). - Eine Beziehung zwischen Wärmeaustausch und Strömungswiderstand der Flüssigkeiten. Physikalische Zeitschrift, vol. 11 (1910).

[3] Blasius (H.). - Grenzschichten in Flüssigkeiten mit kleiner Reibung. Zeitschrift $f$. Mathematik u. Physik, vol. 56 , no. 1 (1908).

[4] Schlichting (H.). - Boundary Layer Theory ", Chapter XIV. Mc Graw Hill, New York (1968).

[5] Smith (A. M. O.). - Improves Solutions of the Falkner and Skan Boundary' Layer Equations. S. MF. Fund Paper No. FF-10, Institute of Aeronautrical Sciences, New York (1954).

[6] Parker (J. D.), Boggs (J. H.), and Blick (E. F.). - «Introduction to Fluid Mechanics and Heat Transfer », p. 331. Addison Wesley, New York (1970). 